\title{
Targeting the DNA damage response enhances CD70 CAR-T cell therapy for renal carcinoma by activating the CGAS-STING pathway
}

\author{
Feng Ji ${ }^{1}$, Fan Zhang ${ }^{1}$, Miaomiao Zhang ${ }^{1}$, Kaili Long ${ }^{1}$, Mingyue Xia ${ }^{1}$, Fei Lu' ${ }^{1}$, Enjie Li ${ }^{1}$, Jiannan Chen ${ }^{1}$, Jun Li ${ }^{2}$, \\ Zhengliang Chen ${ }^{2}$, Li Jing ${ }^{2}$, Shaochang Jia ${ }^{3}$, Rong Yang ${ }^{4}$, Zhigang $\mathrm{Hu}^{1^{*}}$ (10 and Zhigang Guo ${ }^{*^{*}}$
}

\begin{abstract}
Chimeric antigen receptor T-cell (CAR-T) therapy has shown tremendous success in eradicating hematologic malignancies. However, this success has not yet been extrapolated to solid tumors due to the limited infiltration and persistence of CAR-T cells in the tumor microenvironment (TME). In this study, we screened a novel anti-CD70 sCFV and generated CD70 CAR-T cells that showed effective antitumor functions against $\mathrm{CD}^{+} 0^{+}$renal carcinoma cells (RCCs) both in vitro and in vivo. We further evaluated the effect and explored the molecular mechanism of a PARP inhibitor (PARPi) in CAR-T cell immunotherapy by administering the PARPi to mouse xenografts model derived from human RCC cells. Treatment with the PARPi promoted CAR-T cell infiltration by stimulating a chemokine milieu that promoted CAR-T cell recruitment and the modulation of immunosuppression in the TME. Moreover, our data demonstrate that PARPi modulates the TME by activating the CGAS-STING pathway, thereby altering the balance of immunostimulatory signaling and enabling low-dose CAR-T cell treatment to induce effective tumor regression. These data demonstrate the application of CD70 CAR-T cell therapeutic strategies for RCC and the cross-talk between targeting DNA damage responses and antitumor CAR-T cell therapy. These findings provide insight into the mechanisms of PARPis in CAR-T cell therapy for RCC and suggest a promising adjuvant therapeutic strategy for CAR-T cell therapy in solid tumors.
\end{abstract}

Keywords: CAR, CD70, RCC, PARP, Renal carcinoma, Tumor microenvironment, cGAS-STING pathway

\section{To the editor,}

Chimeric antigen receptor $\mathrm{T}$ (CAR-T) cell therapy is emerging as a promising treatment and has achieved beneficial effects in cancer patients [1]. However, the application of CAR-T cell therapy for solid tumors (including renal cell carcinoma, RCC) has been hampered by numerous challenges [2]. It was determined that

\footnotetext{
*Correspondence: huzg_2000@126.com; guo@njnu.edu.cn 1 Jiangsu Key Laboratory for Molecular and Medical Biotechnology, College of Life Sciences, Nanjing Normal University, Nanjing 210023, China

Full list of author information is available at the end of the article
}

hematologic malignancies and solid tumors, including $\mathrm{RCC}$, can constitutively overexpress CD70, which was shown to be an effective target of CAR-T cells in vitro and in vivo [3]. PARP inhibitors (PARPis), the cancer therapeutic agents targeting poly (ADP-ribose) polymerases (PARPs), which play a key role in the DNA damage repair process, have been reported to increase the recruitment of $\mathrm{CD}^{+} \mathrm{T}$ cells to the TME in mouse xenografts [4]. Here, we generated CD70 CAR-T cells with a novel efficient anti-CD70 scFv, and further investigated the effects and mechanisms of PARPi in regulating CAR-T cell therapy in RCC.

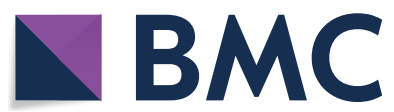

(c) The Author(s) 2021. Open Access This article is licensed under a Creative Commons Attribution 4.0 International License, which permits use, sharing, adaptation, distribution and reproduction in any medium or format, as long as you give appropriate credit to the original author(s) and the source, provide a link to the Creative Commons licence, and indicate if changes were made. The images or other third party material in this article are included in the article's Creative Commons licence, unless indicated otherwise in a credit line to the material. If material is not included in the article's Creative Commons licence and your intended use is not permitted by statutory regulation or exceeds the permitted use, you will need to obtain permission directly from the copyright holder. To view a copy of this licence, visit http://creativecommons.org/licenses/by/4.0/. The Creative Commons Public Domain Dedication waiver (http://creativeco mmons.org/publicdomain/zero/1.0/) applies to the data made available in this article, unless otherwise stated in a credit line to the data. 
Initially, we confirmed the elevated and specific expression of CD70 in RCC as well as in kidney cancer 786-0, A498, and 769-P cells, which paralleled the poor survival prognosis for renal cancer patients with high CD70 expression (Additional file 1: Fig. S1a-e). This result suggested that CD70 might be an effective target for CAR-T cell immunotherapy in RCC [3]. A second-generation humanized CAR was designed with a novel highly efficient anti-CD70 scFv that was identified from a human
scFv phage display library, and the CAR sequence was subcloned into a lentivirus vector (Fig. 1a and Additional file 1: Fig. S2a-d). The percentage of stabilized positive cells was approximately $76 \%$ after infection for 12 days, and the defined $\mathrm{CD} 4 / \mathrm{CD} 8 \mathrm{~T}$-cell ratio was 1:1 (Additional file 1: Fig. S2e-h) [5]. The CD70 expression was undetectable in both CD70 CAR-T cells and inactivated $\mathrm{T}$ cells, whereas it was detected in the CD3/CD28 antibody-activated $\mathrm{T}$ cells (Additional file 1: Fig. S2i, j).

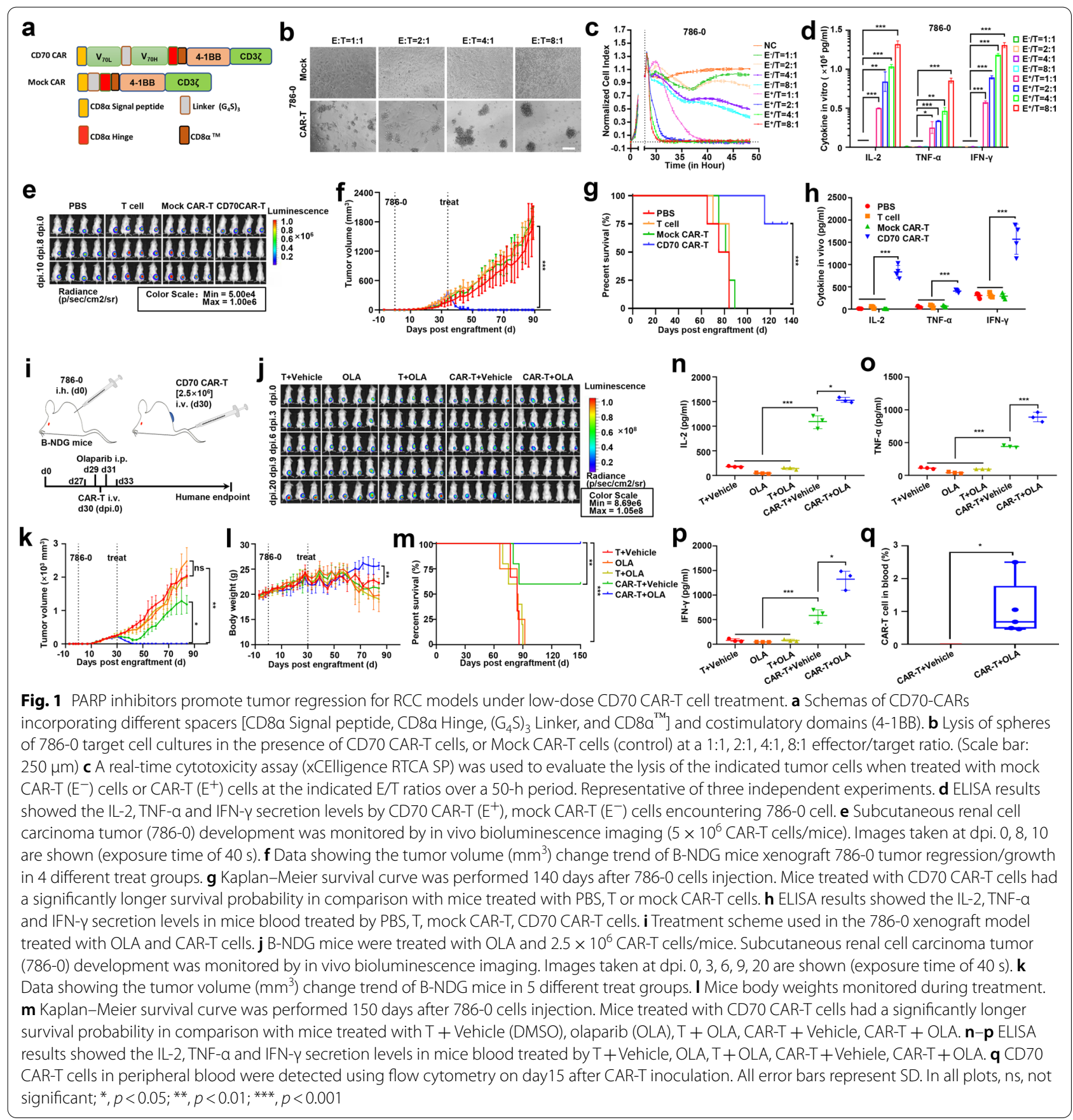




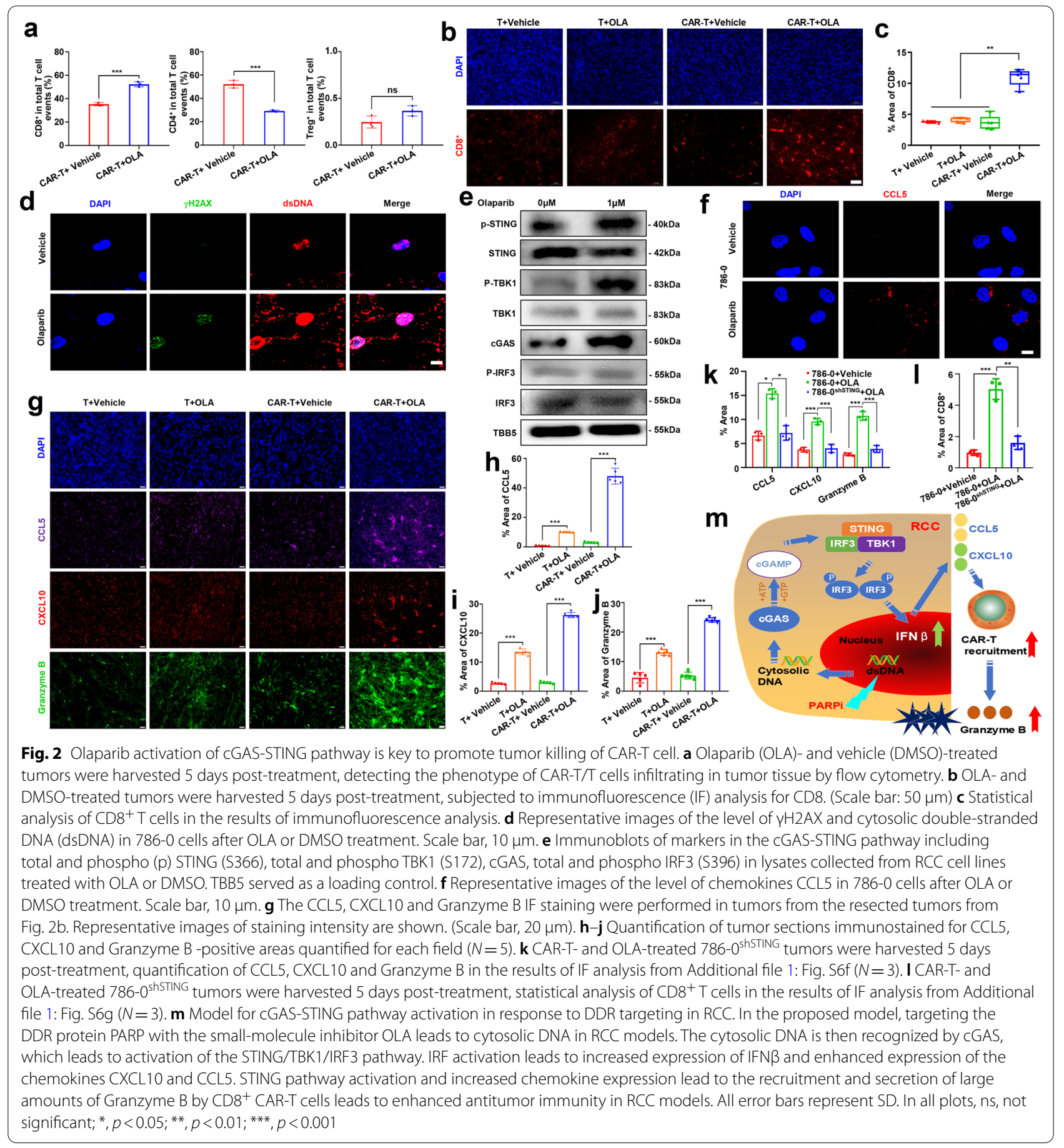

Next, we investigated the antitumor activity of our CD70 CAR-T cells in vitro and in vivo. CD70 CAR-T cells but not mock CAR-T cells induced significantly more target cell lysis of 786-0, A498, and 769-P cells than of HEK293 control cells (Fig. 1b, c and Additional file 1: Fig. S3a, b). Consistently, an increase in cytokines (IL-2, TNF- $\alpha$, and IFN- $\gamma$ ) was observed in a coculture of CAR-T cells and CD70-positive tumor cells (Fig. 1d and Additional file 1: Fig. S3c). Notably, CD70 CAR-T cells induced a significant lytic effect on HEK293 ${ }^{\mathrm{CD} 70+}$ cells but not on HEK293 cells, highlighting the antigen specificity of the target cell lysis (Additional file 1: Fig. S3d, e). Furthermore, mouse xenografts (derived from 786-0 $0^{\text {luc }+}$ cells) treated with CD70 CAR-T cells showed a 
significantly decreased RCC burden and exhibited longer survival times than mice treated with $\mathrm{PBS}$, $\mathrm{T}$ cells, or mock CAR-T cells (Fig. 1e-g and Additional file 1: Fig. S3f-h). Moreover, higher cytokine (IL-2, TNF- $\alpha$, and IFN- $\gamma$ ) secretion levels were detected in the peripheral blood of mice treated with CD70 CAR-T cells than in the peripheral blood of mice treated with control vehicles (Fig. 1h). These results demonstrated that our CD70 CAR-T cells were effective in treating $\mathrm{CD}^{+} 0^{+} \mathrm{RCCs}$ in vitro and in vivo.

The PARPi olaparib (OLA) has been shown to improve the antitumor efficacy of CAR-T cells in breast cancer [6]. We further evaluated the effect of OLA in CAR-T cell therapy in RCC. We first explored the impact of OLA on CAR-T cells and found that OLA showed little effect on CAR-T cell viability when the OLA concentration was lower than $2.5 \mu \mathrm{M}$ (Additional file 1: Fig. S4a, b). We then pretreated RCC cells with OLA following CAR-T cells incubation to detect the effect of OLA on different RCC cells. Cell apoptosis assays indicated that OLA pretreatment promoted the apoptosis of RCC cells but protected CAR-T cells against apoptosis (Additional file 1: Fig. S4c, d). Then, we designed an experimental scheme using mouse xenograft models treated with OLA and CAR-T cells (Fig. 1i). Notably, compared with the control treatments, the combinational treatment of OLA and CD70 CAR-T cells (CAR-T + OLA) showed more effective repression of RCC xenografts, which was accompanied by a better survival rate among tumor-bearing mice (Fig. $1 j-m$ ). The serum levels of TNF- $\alpha$, IL-2, and IFN- $\gamma$ were also higher in the CAR-T + OLA group than in the other four groups (Fig. 1n-p). Notably, CAR-T cells were observed on day 15 post CAR-T injection (dpi. 15) in the blood of the CAR-T + OLA group but not in the CAR-T + Vehicle groups (Fig. 1q), which further supported that OLA treatment promoted CAR-T cell persistence. Moreover, multicolor flow cytometry and IHC assays showed that OLA treatment increased the infiltration of $\mathrm{CD} 8{ }^{+} \mathrm{CAR}-\mathrm{T}$ cells but not $\mathrm{CD} 4^{+} \mathrm{CAR}-\mathrm{T}$ cells and Treg cells in the TME 5 days after injection of CAR-T cells (Fig. 2a-c and Additional file 1: Fig. S5a, b).

Finally, to determine the molecular mechanism of OLA-induced cytotoxic CAR-T cell recruitment in the TME, we detected the activation of the cGAS/ STING signaling pathway, which has been reported to be a critical activator in antitumor immune responses and in $\mathrm{CD}^{+}{ }^{+}$T-cell recruitment with PARPi treatment $[7,8]$. Elevated accumulation of cytosolic DNA and the DNA damage marker $\gamma \mathrm{H} 2 \mathrm{AX}$ was detected in 786-0 cells after treatment with OLA (Fig. $2 \mathrm{~d}$ ). The sensors and key regulators in the cGAS/STING pathway, including cGAS, and the phosphorylation of STING, TBK1, and IRF3, were all upregulated in cells treated with OLA, which was accompanied by upregulation of IFN- $\beta$ expression (Fig. 2 e and Additional file 1: Fig. S5c, d) [9]. The upregulation of IFN- $\beta$ paralleled the observed increases in chemokines such as CCL5 and CXCL10, which are key mediators of the chemotaxis of $\mathrm{CD} 8^{+} \mathrm{T}$ lymphocytes (Fig. $2 \mathrm{f}-\mathrm{i}$ and Additional file 1 : Fig. S5e, f) $[9,10]$. The increase in $\mathrm{CD}^{+}$CAR-T cells ultimately enhanced tumor lysis because of their secretion of large amounts of granzyme B (Fig. 2g, j) [11]. Moreover, knockdown of STING impaired the OLAinduced CCL5 and CXCL10 production in 786-0 cells (786-0 $\left.0^{\text {shSTING }}\right)$ as well as in tumor tissues derived from 786-0 $0^{\text {shSTING }}$ cells, indicating that PARP inhibitorinduced proinflammatory cytokine production is mediated through the cGAS-STING pathway (Fig. $2 \mathrm{k}$ and Additional file 1: Fig. S6a-f). Notably, OLA-induced $\mathrm{CD} 8^{+} \mathrm{CAR}-\mathrm{T}$ cell recruitment and granzyme $\mathrm{B}$ expression was abolished in the 786-0 $0^{\text {shSTING }}$ tumor tissues (Fig. 2k, 1 and Additional file 1: Fig. S6f, g). These data demonstrated that OLA-mediated CAR-T infiltration and persistence in TME are dependent on the cGASSTING pathway [12].

In summary, we demonstrated the efficacy of CD70 CAR-T cells in RCC immunotherapy, and PARPi treatment enhanced this immunotherapy by promoting the infiltration of $\mathrm{CD}^{+}$CAR-T cells in the TME by activating the cGAS/STING signaling pathway (Fig. $2 \mathrm{~m}$ ). This study indicates that the combination of CAR-T cell therapy with PARPi represents a potential therapeutic approach for solid tumors.

\section{Abbreviations}

CAR-T: Chimeric antigen receptor T-cell; TME: Tumor microenvironment; PARP: Poly ADP-ribose polymerase; RCC: Renal cell carcinoma; PARPi: PARP inhibitors; DC: Dendritic cells; OLA: Olaparib; PBMCs: Peripheral blood mononuclear cells; GFP: Green fluorescent protein; scFv: Single-chain variable fragment.

\section{Supplementary Information}

The online version contains supplementary material available at https://doi. org/10.1186/s13045-021-01168-1.

Additional file 1: Supplementary Figures.

Additional file 2: Supplementary materials and methods.

Acknowledgements

We thank every member of the laboratory for helpful comments and suggestions for this manuscript.

\section{Authors' contributions}

FJ, ZG and ZH contributed to the conception and design; FJ, FZ, MZ, KL, MX, EL and JC contributed to the acquisition of data; $J, Z C$ and $L J$ contributed to the analysis and interpretation of data; FJ, FZ, MZ, KL, FL and MX contributed to the writing, review, and/or revision of the manuscript; FJ, FL, SJ, RY, ZG and ZH contributed to the administrative, technical, or material support; ZG and ZH supervised the study. All authors read and approved the final manuscript. 


\section{Funding}

This study was supported by the National Natural Science Foundation of China (81872284) and the Priority Academic Program Development of Jiangsu Higher Education Institutions.

\section{Availability of data and materials}

The authors declare that all data supporting the results in this study are available within the paper and its supplementary information. Source data for the figures in this study are available from the corresponding author upon reasonable request.

\section{Declarations}

\section{Ethics approval and consent to participate}

All in vivo animal experiments were approved by the Committee on the Ethics of Animal Experiments of Nanjing Normal University.

\section{Consent for publication}

Not applicable.

\section{Competing interests}

The authors have no conflicts of interest to declare.

\section{Author details}

${ }^{1}$ Jiangsu Key Laboratory for Molecular and Medical Biotechnology, College of Life Sciences, Nanjing Normal University, Nanjing 210023, China. ${ }^{2}$ Nanjing Blue Shield Biotechology Co., Ltd., Nanjing 210023, China. ${ }^{3}$ Jinling Hospital of Nanjing University, Nanjing 210002, China. ${ }^{4}$ Department of Urology, Nanjing Drum Tower Hospital, Nanjing 210008, China.

Received: 18 May 2021 Accepted: 14 September 2021

Published online: 23 September 2021

\section{References}

1. Sauter CS, Senechal B, Riviere I, et al. CD19 CAR T cells following autologous transplantation in poor-risk relapsed and refractory B-cell non-Hodgkin lymphoma. Blood. 2019;134:626-35.
2. Lesch S, Benmebarek MR, Cadilha BL, et al. Determinants of response and resistance to CAR T cell therapy. Semin Cancer Biol. 2020;65:80-90.

3. Yang M, Tang X, Zhang Z, et al. Tandem CAR-T cells targeting CD70 and B7-H3 exhibit potent preclinical activity against multiple solid tumors. Theranostics. 2020;10:7622-34.

4. Pantelidou C, Sonzogni O, De Oliveria TM, et al. PARP inhibitor efficacy depends on $\mathrm{CD}^{+}{ }^{+}$T-cell recruitment via intratumoral STING pathway activation in BRCA-deficient models of triple-negative breast cancer. Cancer Discov. 2019;9:722-37.

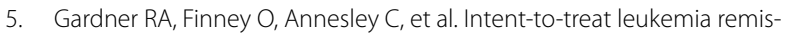
sion by CD19 CART cells of defined formulation and dose in children and young adults. Blood. 2017;129:3322-31.

6. Sun R, Luo H, Su J, et al. Olaparib suppresses MDSC recruitment via SDF1a/CXCR4 axis to improve the anti-tumor efficacy of CAR-T cells on breast cancer in mice. Mol Ther. 2021;29:60-74.

7. Guan J, Lu C, Jin Q, et al. MLH1 deficiency-triggered DNA hyperexcision by exonuclease 1 activates the cGAS-STING pathway. Cancer Cell. 2021;39:109-21.

8. Li W, Lu L, Lu J, et al. CGAS-STING-mediated DNA sensing maintains CD8 ${ }^{+}$ T cell stemness and promotes antitumor T cell therapy. Sci Transl Med. 2020;12:y9013.

9. Sen $\mathrm{T}$, Rodriguez $\mathrm{BL}$, Chen $\mathrm{L}$, et al. Targeting DNA damage response promotes antitumor immunity through STING-mediated T-cell activation in small cell lung cancer. Cancer Discov. 2019;9:646-61.

10. Chen Q, Sun L, Chen ZJ. Regulation and function of the cGAS-STING pathway of cytosolic DNA sensing. Nat Immunol. 2016;17:1142-9.

11. Liu Y, Fang $Y$, Chen $X$, et al. Gasdermin E-mediated target cell pyroptosis by CART cells triggers cytokine release syndrome. Sci Immunol. 2020;5.

12. Xu N, Palmer DC, Robeson AC, et al. STING agonist promotes CAR T cell trafficking and persistence in breast cancer. J Exp Med. 2021:218:e20200844.

\section{Publisher's Note}

Springer Nature remains neutral with regard to jurisdictional claims in published maps and institutional affiliations.

Ready to submit your research? Choose BMC and benefit from:

- fast, convenient online submission

- thorough peer review by experienced researchers in your field

- rapid publication on acceptance

- support for research data, including large and complex data types

- gold Open Access which fosters wider collaboration and increased citations

- maximum visibility for your research: over 100M website views per year

At $\mathrm{BMC}$, research is always in progress.

Learn more biomedcentral.com/submissions 